\title{
Lesão de superfície ocular em unidade de terapia intensiva: ensaio clinico auto-pareado
}

Ocular surface injuries at an intensive care unit: a self-paired clinical trial Lesiones de la superficie ocular en unidad de cuidados intensivos: ensayo clínico autocontrolado

Liliana Yukie Hayakawa ${ }^{1}$ io https://orcid.org/0000-0002-8168-6707

Laura Misue Matsuda ${ }^{1}$ ic https://orcid.org/0000-0002-4280-7203

Kelly Cristina Inoue ${ }^{1}$ iD https://orcid.org/0000-0002-7709-9817

Emerson Kenji Oyamaguchi' ${ }^{1}$ iD https://orcid.org/0000-0003-3626-4780

Ednaldo Ribeiro ${ }^{1}$ id https://orcid.org/0000-0002-4005-5108

Como citar:

Hayakawa LY, Matsuda LM, Inoue KC, Oyamaguchi EK, Ribeiro E. Lesão de superfície ocular em unidade de terapia intensiva: ensaio clinico auto-pareado. Acta Paul Enferm. 2020;33:eAPE20180279.

DOI

http://dx.doi.org/10.37689/ acta-ape/2020A00279

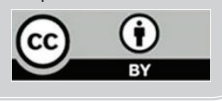

Descritores

Lesões da córnea; Cuidados de enfermagem; Fatores de risco; Unidades de terapia intensiva

Keywords

Corneal injuries; Nursing care; Risk factors;

Intensive care units

Descriptores

Lesiones de la córnea; Atención de enfermeira; Fatores de riesgo; Unidades de cuidados intensivos

Submetido 13 de Novembro de 2018

Aceito

10 de Setembro de 2019

Autor correspondente

Liliana Yukie Hayakawa

E-mail: ilihayakawa@hotmail.com

\section{Resumo}

Objetivo: Analisar a ocorrência de lesão de superfície ocular em Unidade de Terapia Intensiva, entre pacientes sedados e/ou incapazes de piscar.

Métodos: Ensaio Clínico Auto-pareado, realizado na Unidade de Terapia Intensiva de um Hospital da região noroeste do Paraná, Brasil, entre julho/2016 e janeiro/2017. Participaram 27 pacientes sem lesões oculares prévias, submetidos à limpeza ocular bilateral, com solução fisiológica 0,9\% e oclusão do olho direito com filme transparente de poliuretano. Testou-se associação da ocorrência de lesão ocular com variáveis demográficas, clínicas, assistenciais e ambientais, por meio do Teste Exato de Fischer e; comparação do tempo livre de lesão (dias), com e sem uso da cobertura para proteção ocular, por meio do Teste de Wilcoxon.

Resultados: A lesão ocular associou-se com jejum ( $p=0,0039)$, menor risco de morte $(p=0,0056)$ e; maior tempo de internação ( $p=0,0088)$. A oclusão com filme transparente de poliuretano no olho direito foi considerada fator de proteção $(p=0,0019)$, com maior tempo livre de lesão no olho direito (4,1 dias) se comparado ao olho esquerdo (2,4 dias) ( $p=0,00222)$.

Conclusão: Na UTI investigada, a oclusão ocular apresentou-se efetiva à proteção dos olhos, especialmente entre pacientes em jejum, com maior chance de sobrevivência e tempo de internação prolongada.

\section{Abstract}

Objective: To analyze the occurrence of ocular surface injuries at an intensive care unit in patients who were sedated or unable to blink.

Methods: Self-paired clinical trial carried out at the intensive care unit of a hospital in the northwest region of the state of Paraná, Brazil, between July 2016 and January 2017. Twenty-seven patients who did not have previous ocular injuries, submitted to bilateral eye cleaning with $0.9 \%$ salt solution and occlusion of the right eye with transparent polyurethane film, participated in the study. The association of occurrence of ocular injuries with demographic, clinical, care, and environmental variables was investigated by applying Fisher's exact test, and a comparison of the injury-free time (in days) with and without using the eyeshield was performed by using Wilcoxon test.

Results: Ocular injuries were associated with fasting ( $p=0.0039)$, lower risk of death $(p=0.0056)$, and longer hospital stay $(p=0.0088)$. The occlusion of the right eye with transparent polyurethane film was considered a protection factor ( $p=0.0019)$ and was associated with a longer injury-free time in the right eye ( 4.1 days) in comparison with the result obtained for the left eye (2.4 days) $(p=0.00222)$.

Conclusion: In the analyzed intensive care unit, ocular occlusion proved effective in protecting the eye, especially in patients who were fasting, had a higher probability of surviving, and went through a longer hospital stay. 


\section{Resumen}

Objetivo: Analizar los episodios de lesión de la superficie ocular en Unidad de Cuidados Intensivos, entre pacientes sedados o incapaces de pestañear.

Métodos: Ensayo clínico autocontrolado, realizado en la Unidad de Cuidados Intensivos de un hospital de la región noroeste del estado de Paraná, Brasil, entre julio de 2016 y enero de 2017. Participaron 27 pacientes sin lesiones oculares previas, sometidos a una limpieza ocular bilateral, con solución fisiológica 0,9\% y oclusión del ojo derecho con apósito transparente de poliuretano. Se examinó la relación de los episodios de lesión ocular con variables demográficas, clínicas, asistenciales y ambientales, por medio de la Prueba exacta de Fisher, y la comparación del tiempo sin lesión (días), con y sin uso de la cobertura para protección ocular, por medio de la Prueba de Wilcoxon.

Resultados: La lesión ocular se asoció con ayuno ( $p=0,0039)$, menor riesgo de muerte $(p=0,0056)$ y mayor tiempo de internación ( $p=0,0088)$. La oclusión con apósito transparente de poliuretano en el ojo derecho fue considerada factor de protección ( $p=0,0019)$, con un mayor tiempo sin lesión en el ojo derecho (4,1 días) comparado con el ojo izquierdo (2,4 días) ( $p=0,00222)$.

Conclusión: En la UCI investigada, la oclusión ocular se mostró efectiva para la protección de los ojos, especialmente en pacientes en ayuno, con mayor chance de supervivencia y tiempo de internación prolongado.

\section{Introdução}

O avanço tecnológico e a facilidade na disseminaçáo do conhecimento têm proporcionado discussóes e melhorias nos serviços de saúde, mediante a universalização e a padronização de condutas assistenciais, que culminam em maior segurança do paciente e qualidade do atendimento. ${ }^{(1)}$ Nesse sentido, grande parte dos esforços de instituições hospitalares, impulsionadas por regulamentação específica, têm se voltado à implementação de protocolos básicos, consonantes com as metas internacionais de segurança do paciente. ${ }^{(2)}$

Reconhece-se, no entanto, que a segurança do paciente não é construída apenas por melhorias nas áreas mais problemáticas da assistência à saúde. Especialmente em ambientes complexos, onde podem existir erros, um desafio importante no campo da segurança do paciente é evitar danos decorrentes da assistência, priorizando-se todos e cada um dos pacientes. ${ }^{(3)}$

Dentre os ambientes de maior complexidade assistencial, destaca-se a Unidade de Terapia Intensiva (UTI), onde a padronizaçáo de condutas tem se sustentado em diretrizes terapêuticas que alertam para o manejo clínico de condiçôes e agravos de alto impacto sobre a mortalidade dos pacientes. ${ }^{(4,5)}$ Ao exemplo disso, tem-se estabelecido cuidados para a prevenção de pneumonia associada à ventilação mecânica, aos distúrbios hidroeletrolíticos, à iminência dos estados de choque, dentre outras patologias emergentes. ${ }^{(6)}$

Apesar de conter açôes transversais e multiprofissionais, pautadas nas melhores evidências disponíveis, as diretrizes de práticas clínicas se configuram como ferramentas úteis à provisão dos melhores cui- dados aos pacientes, por auxiliar em essência, a decisão médica. ${ }^{(7)}$ Desse modo, atividades estreitamente relacionadas à Enfermagem, tais como os cuidados para proteção ocular, são relegadas a segundo plano e assim, comumente não constam em protocolos de $\mathrm{UTI} ;^{(6)}$ seja pela falta de conhecimento do enfermeiro e da equipe multidisciplinar a acerca da anatomia e fisiologia, como pelo desconhecimento da forma adequada de avaliação ocular e dos cuidados a serem implementados. ${ }^{(8,9)}$

Pacientes internados em UTI, frequentemente se encontram sob risco de lesão na córnea devido à sedação, coma, ventilação mecânica e medicação. ${ }^{(9-13)}$ Nessas condiçóes, o fechamento palpebral incompleto produz ressecamento da mucosa da superfície e de todos os tecidos epiteliais da córnea, resultando em ulceração ou ceratopatia de exposição, ${ }^{(8,10,14,15)}$ cuja incidência pode atingir até $83,3 \%{ }^{(8)}$

No cenário mundial, para reduzir a incidência e a prevalência de alteraçôes da superfície ocular em pacientes críticos, diferentes intervenções têm sido instituídas à prevenção deste agravo e para isso, têm sido utilizados diferentes tipos de coberturas, colírios e pomadas. ${ }^{(16-18)}$

Dentre os recursos utilizados na prevençáo de lesão de superfície ocular em UTI, a aplicação de cobertura de polietileno tem apresentado discreta superioridade ao ser comparada com outros produtos para proteçáo ocular. ${ }^{(18,19)}$ Contudo, o filme de polietileno não se encontra aprovado como produto para saúde no Brasil, mas similarmente tem-se disponível para aplicação tópica em seres humanos o filme transparente de poliuretano com polímeros de etileno. ${ }^{(20)}$

No tocante à realidade brasileira, os cuidados oculares durante a assistência de enfermagem são li- 
mitados porque, de acordo com a literatura, há falta de consenso e de padronização. ${ }^{(21)}$ Com isso, as ações de enfermagem são ainda direcionadas por experiências individuais, adaptadas aos contextos locais.

De modo a garantir a segurança da assistência de enfermagem em UTI, faz-se importante aprofundar o conhecimento sobre fatores de risco para a ocorrência de lesão ocular, aliado a estudos das tecnologias para a sua prevenção. Mediante a isso, o objetivo deste estudo consistiu em analisar a ocorrência de lesão de superfície ocular em UTI, entre pacientes sedados e/ou incapazes de piscar.

\section{Métodos}

Ensaio Clínico Auto-pareado, referente à prevenção de lesão de superfície ocular, por oclusão com filme transparente de poliuretano, realizado entre julho de 2016 a janeiro de 2017, na UTI de um Hospital da região noroeste do Paraná, Brasil. A UTI referida, dispóe de sete leitos de internação, é credenciada para atendimento referenciado de pacientes do Sistema Único de Saúde (SUS). O hospital atende também às demandas particulares e de operadoras de plano privado de saúde.

Foi realizada amostra não probabilística, com avaliação de elegibilidade de todos os pacientes admitidos na UTI, incluindo-se aqueles que atenderam aos seguintes critérios: ter 14 anos ou mais; possuir Escala de Richmond de Agitação-Sedação (RASS) menor ou igual a -4 pontos (quatro negativo) ou com ausência do reflexo de piscar, a partir do momento da admissão na UTI. Como critérios de exclusão, foram considerados: existência prévia à admissão na UTI de diferenças anatômicas entre os olhos; extirpação ocular ou; presença de alteraçóes que indicassem lesão de superfície ocular (derrame ocular, ulceração, hiperemia, edema, secreção, pápulas e/ou opacificação corneana), confirmados por meio do exame clínico e teste com fluoresceína sódica; análise de secreção ocular com cultura; impossibilidade de visualização e avaliação da superfície ocular (edema palpebral/periorbital acentuado) ou; não aceite formal do familiar ou representante legal à participação na pesquisa.
De 143 pacientes elegíveis, 116 foram excluídos (92 por não atender a algum critério de inclusão; 5 por não assinar o consentimento livre e esclarecido; 18 por apresentar lesão ocular no momento da avaliação inicial e; 1 devido a cultura de secreção ocular positiva). Desse modo, a pesquisa contou com 27 participantes, os quais foram submetidos à limpeza diária de ambos os olhos com soro fisiológico 0,9\% e gaze estéril, bem como oclusão apenas do olho direito com fração de filme transparente de poliuretano (Tegaderm ${ }^{\text {тм }}$ Rolo Filme Transparente não estéril, $15 \mathrm{~cm}$ x $10 \mathrm{~m}, 3 \mathrm{M}$, Brasil).

Os valores e dados referentes a caracterização demográfica e clínica no momento da inclusão do participante e, diariamente em relação à evolução clínica, à avaliação ocular, informações do ambiente e da assistência de enfermagem, foram compilados, tratados eletronicamente e submetidos à estatística descritiva e inferencial.

Os participantes que desenvolveram lesão de superfície ocular em um ou ambos os olhos foram alocados em um grupo $(n=13)$ e aqueles que não desenvolveram lesão de superfície ocular $(n=14)$ foram alocados em outro grupo.

Para comparação do grupo com e sem lesão de superfície ocular, realizou-se o Teste Exato de Fischer no Software Epiinfoâ, com nível de significância estabelecido em 5\%, considerando-se as seguintes variáveis: sexo; idade (ponto de corte 50 anos); tempo de internação na UTI (ponto de corte 7 dias); média da pontuação APACHE II; média da pontuação RASS; média da pontuação SOFA; média de PEEP; comprometimento cardíaco, neurológico, pulmonar, renal e endócrino; sepse; modo ventilatório; uso de analgo-sedação e droga vasoativa; jejum; condição de alta; lesão no olho direito; e umidade e temperatura ambiente.

Também se procedeu ao Teste de Wilcoxon $(\mathrm{n}=27 \mathrm{x} 2)$, no programa SPSS ${ }^{\hat{a}}$ Statistics $21^{\hat{a}}$, com nível de significância estabelecido em 5\%, para comparação do tempo livre de lesão (dias) no olho direito e esquerdo.

Ressalta-se que, todas as exigências éticas e legais $^{(20,22)}$ foram cumpridas e, no decorrer da pesquisa, as alteraçóes oculares detectadas foram imediatamente reportadas ao intensivista, para que o diag- 
nóstico fosse confirmado e o processo terapêutico, iniciado. Esta pesquisa se encontra registrada sob Parecer Favorável nº 1.601.549/2016 do Comitê de Ética da Faculdade Ingá, da cidade de MaringáPR, e sob o Registro Brasileiro de Ensaios Clínicos (ReBEC) no. UTN U1111-1191-2395.

\section{Resultados}

Dentre os 27 participantes, 18 (66,7\%) eram mulheres e $9(33,3 \%)$ eram homens, cuja idade variou de 22 a 101 anos, com média de 72,3 anos (desvio padrão=15,6 anos). Destes, $13(48,1 \%)$ foram alocados no grupo lesão de superfície ocular e 14 $(51,9 \%)$ no grupo sem lesão de superfície ocular.

Destaca-se que, dentre os 13 pacientes que apresentaram lesão de superfície ocular, todos apresentaram algum sinal comprometimento, ainda que inicial, do olho esquerdo e, apenas $6(46,2 \%)$ do olho direito (protegido com fração de filme transparente de poliuretano).

As variáveis demográficas (sexo, idade e raça), ventilatórias (modo ventilatório e PEEP), de comprometimento de sistema orgânico (cardíaco, neurológico, pulmonar, renal e endócrino), uso de drogas (analgo-sedação e droga vasoativa) não apresentaram associação significativa com o desenvolvimento de lesão de superfície ocular e, as demais variáveis testadas constam na tabela 1 .

Os dados da comparação do tempo livre de lesão (dias) entre os olhos direito (ocluído com filme transparente de poliuretano) e olhos esquerdo (sem oclusão de proteção) está disposta na tabela 2 .

\section{Discussão}

O presente estudo se limita a uma realidade local e de avaliação de uma única intervenção protetora, com rigor no controle de variáveis relacionadas ao paciente, determinada pela própria metodologia de alocação para intervenção do estudo primário.

Com vistas à segurança do paciente e à qualidade do cuidado, espera-se que pesquisas como esta, que aprofunda o conhecimento sobre fatores de risco e estratégias de prevenção de lesão de superfície ocular
Tabela 1. Associação de variáveis com o desenvolvimento de lesão de superfície ocular em pacientes internados em UTI

\begin{tabular}{|c|c|c|c|}
\hline & Lesão & Sem lesão & $p$-value \\
\hline Variáveis & $n(\%)$ & $\mathrm{n}(\%)$ & \\
\hline \multicolumn{4}{|l|}{ Sexo } \\
\hline Feminino & $9(69,2)$ & $9(64,3)$ & \\
\hline Masculino & $4(30,8)$ & $5(35,7)$ & 0,5545 \\
\hline \multicolumn{4}{|l|}{ Idade (anos) } \\
\hline$<50$ & $2(15,4)$ & $2(14,2)$ & \\
\hline$\geq 50$ & $11(84,6)$ & $12(85,8)$ & 0,6733 \\
\hline \multicolumn{4}{|l|}{ Sepse } \\
\hline Sim & $8(61,5)$ & $9(64,3)$ & \\
\hline Não & $5(38,5)$ & $5(35,7)$ & 0,5982 \\
\hline \multicolumn{4}{|l|}{ Jejum } \\
\hline Sim & $13(100)$ & $7(50)$ & \\
\hline Não & - & $7(50)$ & 0,0039 \\
\hline \multicolumn{4}{|l|}{ RASS (pontos) } \\
\hline$>-2,7$ & $8(61,5)$ & $7(50)$ & \\
\hline$\leq-2,7$ & $5(38,5)$ & $7(50)$ & 0,4788 \\
\hline \multicolumn{4}{|l|}{ APACHE II (pontos) } \\
\hline$<35,8$ & $10(76,9)$ & $6(42,9)$ & \\
\hline$\geq 35,8$ & $3(23,1)$ & $8(57,1)$ & 0,0789 \\
\hline \multicolumn{4}{|l|}{ Risco de morte $(\%)$} \\
\hline$<70,4$ & $10(76,9)$ & $3(21,4)$ & \\
\hline$\geq 70,4$ & $3(23,1)$ & $11(78,6)$ & 0,0056 \\
\hline \multicolumn{4}{|l|}{ SOFA (pontos) } \\
\hline$<10,0$ & $8(61,5)$ & $4(28,6)$ & \\
\hline$\geq 10,0$ & $5(38,5)$ & $10(71,4)$ & 0,0906 \\
\hline \multicolumn{4}{|c|}{ Tempo de internação na UTI (dias) } \\
\hline$<7$ & $1(7,7)$ & $8(57,1)$ & \\
\hline$\geq 7$ & $12(92,3)$ & $6(42,9)$ & 0,0088 \\
\hline \multicolumn{4}{|c|}{$\begin{array}{l}\text { Temperatura do ambiente de momento } \\
\left({ }^{\circ} \mathrm{C}\right)\end{array}$} \\
\hline$<22,9$ & $5(38,5)$ & $9(64,3)$ & \\
\hline$\geq 22,9$ & $8(61,5)$ & $5(35,7)$ & 0,1697 \\
\hline \multicolumn{4}{|c|}{ Umidade do ambiente de momento (\%) } \\
\hline$<55,8$ & $9(69,2)$ & $9(64,3)$ & \\
\hline$\geq 55,8$ & $4(30,8)$ & $5(35,7)$ & 0,5545 \\
\hline \multicolumn{4}{|l|}{ Condição de alta } \\
\hline Sobrevivente & $7(53,8)$ & $3(21,4)$ & \\
\hline Não sobrevivente & $6(46,2)$ & $11(78,6)$ & 0,0891 \\
\hline \multicolumn{4}{|c|}{ Lesão no olho direito** } \\
\hline Sim & $7(53,8)$ & - & \\
\hline Não & $6(46,2)$ & $14(100)$ & 0,0019 \\
\hline
\end{tabular}

*Risco de morte estimado pelo APACHE II; ** Olho ocluído com filme transparente de poliuretano para prevenção de lesão de superfície ocular

Tabela 2. Comparação do tempo livre de lesão (dias), entre olhos direitos e esquerdos de pacientes internados em UTI

\begin{tabular}{lccccccc}
\hline Dias livre de lesão & $\mathbf{n}$ & Média & $\begin{array}{c}\text { Desvio } \\
\text { padrão }\end{array}$ & Mínimo & Máximo & $p$-value \\
\hline Olho direito* & 27 & 4,1 & 3,2 & 1,0 & 12,0 & 0,00222 \\
Olho esquerdo & 27 & 2,4 & 1,0 & 1,0 & 6,0 & \\
\hline
\end{tabular}

*Olho ocluído com filme transparente de poliuretano para prevenção de lesão de superfície ocula

em pacientes de UTI, possam contribuir à elaboração e implementação de protocolos assistenciais voltados aos cuidados com os olhos. Além disso, os resultados aqui obtidos podem subsidiar a tomada de decisão 
por gestores e profissionais acerca da incorporação do filme transparente de poliuretano para proteção dos olhos de pacientes sedados ou incapazes de piscar, ventilados mecanicamente. $\mathrm{O}$ fomento em discussóes sobre o tema no campo do ensino e da prática em saúde também pode ser considerado.

Reconhecidamente, a sedação, coma, ventilação mecânica e medicação são fatores de risco associados ao desenvolvimento de lesão de superfície ocular entre pacientes internados em UTI. ${ }^{(10-14,22)}$ Nesse aspecto, tanto a modalidade ventilatória (sincronizada ou controlada) como o escore de RASS ( -4 ou -5 pontos) correspondem a níveis diferenciados de resposta do paciente e podem refletir a capacidade de piscar os olhos, cujo grau de prejuízo não foram associados ao desenvolvimento de lesão de superfície ocular (Tabela 1).

Embora o jejum tenha se associado ao desenvolvimento de lesão de superfície ocular $(\mathrm{p}=0,0039)$, pelo fato de a oferta de nutrientes ser essencial à manutenção da integridade e viabilidade tissular, ${ }^{(23,24)}$ não foi constatada influência isolada de nenhum sistema orgânico (cardíaco, neurológico, pulmonar, renal e endócrino) na perfusão e/ou manutenção da integridade ocular. Isso porque, o funcionamento do corpo humano é complexo e os sistemas orgânicos interagem entre si. Ademais, apesar da falta de oxigênio estar relacionada tradicionalmente à falência renal, hepática, pulmonar e cardiocirculatória é preciso considerar, adicionalmente, a relação de outros dois sistemas orgânicos muito dinâmicos e interdependentes, o endotelial e o sanguíneo. ${ }^{(25)}$

Destaca-se que, tanto o risco de morte estimado pelo APACHE II ( $\mathrm{p}=0,0056)$ como o tempo de internação na UTI ( $p=0,0088$ ) se associaram ao desenvolvimento de lesão de superfície ocular (Tabela 1), conforme constam em estudos semelhantes. ${ }^{(8,10,26)}$ Desse modo, pacientes com menor risco de morte $(n=10$, $76,9 \%)$ e maior permanência na UTI $(n=12,92,3 \%)$ apresentaram maior proporção de lesão de superfície ocular (Tabela 1); afinal, a sobrevivência e o prolongamento da internação promoveram maior tempo de exposição aos fatores intrínsecos e extrínsecos, contribuindo ao desenvolvimento da lesão ocular.

Verifica-se ainda, na tabela 1 , que o olho direito esteve associado à não ocorrência de lesão de superfície ocular ( $\mathrm{p}=0,0019)$. Sobre isso, é importante destacar que todos os olhos direito foram protegidos por oclusão com filme transparente de poliuretano, o qual contribuiu com quase o dobro de tempo livre de lesão ocular quando comparado ao olho esquerdo que náo foi protegido ( $\mathrm{p}=0,00222$ ) (Tabela 2).

Ao considerar que o desenho do estudo primário (Ensaio Clínico Auto-Pareado) garantiu o controle de variáveis associadas ao paciente, com redução de bias intrínsecas, a proteção ocular aqui identificada atribui-se ao uso de oclusão com filme transparente de poliuretano. Assim, considera-se que a cobertura utilizada auxilia na manutenção mecânica da umidade dos olhos e evita o ressecamento por fatores externos/ambientais, de modo similar à cobertura transparente de polietileno, que tem sido apontada internacionalmente como estratégia efetiva na proteção de lesão de superfície ocular em UTI. ${ }^{(8,26,27)}$

Sabe-se que, fatores ambientais, como a temperatura e umidade relativa do ar podem exercer influência sobre as características do filme lacrimal e na taxa de evaporação da lágrima, pois a exposição prolongada a temperaturas adversas poderia resultar em sintomas de olho seco e, finalmente, em alteraçôes da superfície ocular. ${ }^{(28,29)}$

Neste estudo, a média da temperatura ambiente de momento $\left(22,9^{\circ} \mathrm{C}\right)$ e de umidade relativa do ar de momento $(55,8 \%)$ náo se associaram ao desenvolvimento de lesão de superfície ocular. Isso porque, as condiçóes ambientais do local do estudo foram mantidas tal como a normatização de condiçôes de conforto e controle higrotérmico em UTI, com sistema de refrigeração para garantia de manutençáo de temperatura e umidade entre 21 a $24^{\circ} \mathrm{C}$ e entre 40 e $60 \%$, respectivamente, ${ }^{(30)}$ o que reforça o pressuposto de que, independentemente da temperatura ou umidade, é a exposição ao ambiente (olho esquerdo) ou náo (olho direito), que influenciou o desenvolvimento de lesão de superfície ocular.

Considera-se, portanto, que a implementação de proteção ocular com filme transparente de poliuretano é uma estratégia de cuidado de enfermagem em UTI, que contribui à redução deste tipo de agravo e de suas complicaçóes. Para isso, é fundamental que sejam investidos esforços na capacitação das equipes e aquisição de recursos que garantam a proteção ocular por oclusão com filme transparente de poliuretano, como parte dos protocolos assistenciais em UTI. 


\section{Conclusão}

A estratificação de fatores de risco reconhecidos para o desenvolvimento de lesão de superfície ocular entre pacientes sedados ou com piscar dos olhos prejudicados e ventilados mecanicamente em UTI, não são, em sua maioria, significativos quando analisados de maneira isolada. Apesar disso, de acordo com os resultados, conclui-se que, o uso de filme transparente de poliuretano apresenta-se como estratégia efetiva para a proteção dos olhos em UTI, especialmente entre aqueles mantidos sob jejum, com maior possibilidade de sobrevivência e tempo de internação prolongada.

\section{Colaborações}

\section{Hayakawa LY, Matsuda LM, Inoue KC, Oyamaguchi} EK e Ribeiro E contribuíram com a concepção do projeto, análise e interpretação dos dados, redação do artigo, revisão crítica relevante do conteúdo intelectual e aprovação final da versão a ser publicada.

\section{Referências}

1. Almeida Q, Fófano GA. Tecnologias leves aplicadas ao cuidado de enfermagem na unidade de terapia intensiva: uma revisão de literatura. HU Rev. 2016;42(3):191-6.

2. Agência Nacional de Vigilância Sanitária (ANVISA). Resolução RDC no. 36, de 25 de julho de 2013. Brasília (DF): ANVISA; 2013.

3. World Health Organization (WHO). Patient safety: Making health care safer. Geneva: WHO; 2017.

4. Nogueira JJ, Ferreira JA, Albuquerque AM, Agra G. Fatores agravantes e atenuantes à percepção de morte em UTI: a visão dos pacientes. Rev Fund Care Online. 2017;9(1):51-6.

5. Silva CR, Abrão FM, Oliveira RC, Louro $T Q$, Moura LF, Silva RC. Representações sociais de enfermeiros sobre o processo de morte e morrer em UTI. Cienc Cuid Saude. 2016;15(3):474-81.

6. Kuruvilla S, Peter J, David S, Premkumar PS, Ramakrishna K, Thomas $L$, et al. Incidence and risk factor evaluation of exposure keratopathy in critically ill patients: a cohort study. J Crit Care. 2015;30(2):400-4.

7. Lisboa TC, Cavalcanti AB, Lobo SM. Diretrizes brasileiras em terapia intensiva: vamos encarar este desafio.... Rev Bras Ter Intensiva. 2016;28(3):213-4.

8. Kocaçal Güler E, Eşer I, Eğrilmez S. Effectiveness of polyethylene covers versus carbomer drops (Viscotears) to prevent dry eye syndrome in the critically ill. J Clin Nurs. 2011;20(13-14):1916-22.

9. Werly Alvarenga A, Ercoli FF, Botoni FA, Oliveira JA, Chianca TC. Lesões na córnea: incidência e fatores de risco em unidade de terapia intensiva. Rev Lat Am Enfermagem. 2008;19(5):1088-95.
10. van der Wekken RJ, Torn E, Ros FE, Haas LE. A red eye on the intensive care unit. Exposure keratopathy with corneal abrasion secondary to lagophthalmos due to chemosis. Neth J Med. 2013;71(4):204-7.

11. Alansari MA, Hijazi MH, Maghrabi KA. Making a difference in eye care of the critically ill patients. J Intensive Care Med. 2015;30(6):311-7.

12. Güler EK, Eşer I, Fashafsheh IH. Intensive Care Nurses' Views and Practices for Eye Care: An International Comparison. Clin Nurs Res. 2017;26(4):504-24.

13. Kousha 0 , Kousha Z, Paddle J. Incidence, risk factors and impact of protocolised care on exposure keratopathy in critically ill adults: a twophase prospective cohort study. Crit Care. 2018;22(1):5.

14. Demirel S, Cumurcu T, Firat P, Aydogan MS, Doğanay S. Effective management of exposure keratopathy developed in intensive care units: the impact of an evidence based eye care education programme. Intensive Crit Care Nurs. 2014;30(1):38-44.

15. Davoodabady Z, Rezaei K, Rezaei R. The Impact of Normal Saline on the Incidence of Exposure Keratopathy in Patients Hospitalized in Intensive Care Units. Iran J Nurs Midwifery Res. 2018;23(1):57-60.

16. Araújo D, Ribeiro N, Aleixo-Silva P, Rezende-Macieira T, da Silva P, Machado-Chianca T. Dry eye in critically ill patients: integrative review Olho seco em pacientes críticos: revisão integrativa. Rev Pesqui Cuid Fund Online. 2017; 9(4):907-16.

17. Masoudi Alavi N, Sharifitabar Z, Shaeri M, Adib Hajbaghery M. An audit of eye dryness and corneal abrasion in ICU patients in Iran. Nurs Crit Care. 2014;19(2):73-7.

18. Sivasankar S, Jasper S, Simon S, Jacob P, John G, Raju R. Eyecare in ICU. Indian J Crit Care Med. 2006;10(1):11-4.

19. Zhou Y, Liu J, Cui Y, Zhu H, Lu Z. Moisture chamber versus lubrication for corneal protection in critically ill patients: a meta-analysis. Cornea. 2014;33(11):1179-85.

20. Agência Nacional de Vigilância Sanitária (ANVISA). Resolução - RE $n^{0}$ 1.133, de 01 julho de 2002. Dispõe sobre a concessão de registros, as alterações e as retificações de registros dos produtos para a saúde. Brasília (DF): ANVISA; 2002.

21. Werli-Alvarenga A, Ercole F, Botoni F, Oliveira J, Chianca T. Lesões na córnea: incidência e fatores de risco em Unidade de Terapia Intensiva. Rev Lat Am Enfermagem. 2011;19(5):1088-95.

22. Conselho Nacional de Saúde. Comissão Nacional de Ética em Pesquisa. Resolução n. 466, de 12 de dezembro de 2012. Aprova diretrizes e normas regulamentadoras de pesquisa envolvendo seres humanos [Internet]. Diário Oficial da União, Brasília, DF, n. 2, p. 9, jul. 2012. Seção 1. [citado 2019 Set 12]. Disponível em: <http://conselho.saude. gov.br/resolucoes/2012/Res0466.pdf>

23. Agência Nacional de Vigilância Sanitária (ANVISA). Resolução RDC n 010, de 20 de fevereiro de 2015. Dispõe sobre o regulamento para a realização de ensaios clínicos com dispositivos médicos no Brasil. Brasília (DF): ANVISA; 2015.

24. Sousa Júnior BS, Mendonça AE, Duarte FH, Silva CC. Diagnóstico de enfermagem risco de úlcera por pressão segundo taxonomia II da North American Nursing Diagnosis Association. ESTIMA. 2017;15(4):222-8.

25. Bjerkvig CK, Strandenes G, Eliassen HS, Spinella PC, Fosse TK, Cap AP, et al. "Blood failure" time to view blood as an organ: how oxygen debt contributes to blood failure and its implications for remote damage control resuscitation. Transfusion. 2016;56 Suppl 2:S182-9.

26. So HM, Lee CC, Leung AK, Lim JM, Chan CS, Yan WW. Comparing the effectiveness of polyethylene covers (Gladwrap) with lanolin (Duratears) eye ointment to prevent corneal abrasions in critically ill patients: a randomized controlled study. Int J Nurs Stud. 2008;45(11):1565-71. 
27. Kalhori RP, Ehsani S, Daneshgar F, Ashtarian H, Rezaei M. Different Nursing Care Methods for Prevention of Keratopathy Among Intensive Care Unit Patients. Glob J Health Sci. 2015;8(7):2127.

28. Abusharha AA, Pearce El, Fagehi R. Effect of Ambient Temperature on the Human Tear Film. Eye Contact Lens. 2016;42(5):30812.
29. Abusharha AA, Pearce El. The effect of low humidity on the human tear film. Cornea. 2013;32(4):429-34.

30. Associação Brasileira de Normas Técnicas (ABNT). NBR 7256: 2005, de 30 de março de 2005. 2005. Dispõe sobre o Tratamento de ar em estabelecimentos assistenciais de saúde (EAS) - Requisitos para projeto e execução das instalações. [Internet]. [citado 2019 Set 12]. Disponível em http://www.ductbusters.com.br/normas/NBR_7256.pdf 